\title{
Insulin requirements throughout pregnancy in women with type 1 diabetes mellitus: three changes of direction
}

\author{
A. García-Patterson • I. Gich • S. B. Amini • \\ P. M. Catalano $\cdot$ A. de Leiva $\cdot$ R. Corcoy
}

Received: 30 September 2009 / Accepted: 16 November 2009/Published online: 15 December 2009

(C) Springer-Verlag 2009

\begin{abstract}
Aims/hypothesis The aim of the study was to analyse the insulin requirements of women with type 1 diabetes mellitus throughout pregnancy.

Methods We have examined the weekly mean blood glucose (mmol/l), insulin requirements $\left(\mathrm{U} \mathrm{kg}^{-1} \mathrm{day}^{-1}\right)$ and total insulin requirements (U/day) in 65 women with type 1 diabetes mellitus and tight metabolic control since before pregnancy $\left(\mathrm{HbA}_{1 \mathrm{c}} \leq 6.0 \%\right)$.

Results Both insulin requirement and total insulin requirement displayed a peak in week 9, a nadir in week 16 and a second peak in week 37. For the change in insulin requirement $(4.08 \%$ per week) and in total insulin requirement $(5.19 \%$ per week $)$, the sharpest slope was observed
\end{abstract}

Electronic supplementary material The online version of this article (doi:10.1007/s00125-009-1633-z) contains supplementary material, which is available to authorised users.

\footnotetext{
A. García-Patterson · A. de Leiva $\cdot$ R. Corcoy $(\bowtie)$

Department of Endocrinology and Nutrition,

Hospital de la Santa Creu i Sant Pau,

Sant Antoni $\mathrm{M}^{\mathrm{a}}$ Claret, 167,

Barcelona 08025, Spain

e-mail: rcorcoy@santpau.cat

I. Gich

Department of Clinical Epidemiology,

Hospital de la Santa Creu i Sant Pau,

Barcelona, Spain

S. B. Amini • P. M. Catalano

Department of Reproductive Biology,

Case Western Reserve University, MetroHealth Medical Center, Cleveland, $\mathrm{OH}$, USA

A. de Leiva $\cdot$ R. Corcoy

CIBER Bioengineering, Biomaterials and Nanotechnology, Instituto de Salud Carlos III,

Madrid, Spain

from week 16 to week 37. However, two changes of direction took place in the first 11 weeks and eight out of nine episodes of severe hypoglycaemia requiring treatment with glucagon or i.v. glucose took place in the first 16 weeks.

Conclusions/interpretation Pregnant women with type 1 diabetes mellitus and tight metabolic control since before pregnancy displayed changes in insulin requirement and total insulin requirement with successive changes of direction. The sharpest slope was observed between 16 and 37 weeks, but insulin requirements were more unstable in the first 16 weeks. This information could help patients and physicians to react to changes in glycaemic pattern in a prompt and adequate way.

Keywords Insulin requirements · Pregnancy · Type 1 diabetes mellitus

$\begin{array}{ll}\text { Abbreviations } \\ \text { CBG } & \text { Capillary blood glucose } \\ \text { GA } & \text { Gestational age } \\ \text { IR } & \text { Insulin requirement } \\ \text { MBG } & \text { Mean blood glucose } \\ \text { SMBG } & \text { Self-monitoring of blood glucose } \\ \text { TIR } & \text { Total insulin requirement }\end{array}$

\section{Introduction}

In women with diabetes mellitus, tight metabolic control is important both before and during pregnancy in order to reduce pregnancy complications. The association of poor glycaemic control with untoward perinatal outcome has been established for congenital malformations [1, 2], 
miscarriage [3], perinatal mortality [4], pre-eclampsia [5], increased birthweight [6], neonatal hypoglycaemia [7] and respiratory distress [8], among others.

In addition, maintenance of good glycaemic control is difficult because insulin requirement (IR) is continuously changing. The changes include a decrease in IR in the first trimester $[9,10]$ and an increase in the second half of pregnancy $[9,11,12]$. However, the information offered by these papers is incomplete because glycaemic values during pregnancy are not provided, information is lacking on glycaemic levels in pre-pregnancy and the first weeks of pregnancy and, in most cases, women did not have good glycaemic control before pregnancy. Thus, changes in insulin requirement do not only reflect pregnancy-related alterations but efforts to improve glycaemic control. Such efforts to achieve near-normal glycaemic control together with the effect of changing insulin requirements underlie the high rate of severe hypoglycaemia in pregnant women [10], which can in turn have serious consequences [13]. Thus, the knowledge of the expected course of insulin requirement in pregnant women with type 1 diabetes mellitus would help them to achieve metabolic goals. The observation of a transient increase in IR during early pregnancy in a woman with type 1 diabetes mellitus prompted this study.

\section{Methods}

Patients We conducted a retrospective cohort study of women with type 1 diabetes mellitus attending the prepregnancy clinic. Women were eligible if: pregnancy was single; pre-pregnancy $\mathrm{HbA}_{1 \mathrm{c}}$ was $\leq 6 \%$ (mean $+3 \mathrm{SD}$ of a healthy non-pregnant group); information on insulin doses and self-monitoring of blood glucose (SMBG) was available for 4 weeks immediately prior to pregnancy; and the pregnancy proceeded to $\geq 34$ weeks of gestation.

Type 1 diabetes mellitus was diagnosed clinically. Information on C-peptide and diabetes-related autoantibodies was not always available. Gestational age (GA) was determined from dates and modified after early pregnancy ultrasound if discordance was significant.

Assessment of metabolic control Treatment included isoenergetic diet, SMBG and insulin therapy (multiple injections in 51 patients and insulin pump in 14 patients). Diet was calculated after basal requirements [14] plus estimated exercise requirements. SMBG was performed 6-8 times/day (pre-prandial, $1 \mathrm{~h}$ postprandial, at bedtime and during the night). The targets for capillary blood glucose $(\mathrm{CBG})$ were $3.3-5.0 \mathrm{mmol} / \mathrm{l}$ pre-prandial and 5.5$6.6 \mathrm{mmol} / 1 \mathrm{~h}$ postprandial. Therapy adjustments were performed both by the patient and medical staff whenever glycaemic targets in a period of the day were not met on two consecutive days. Modifications included not only insulin dose but distribution of carbohydrate and time interval between insulin dose and meal. Pregnancy did not imply a therapeutic change unless dictated by CBG. At 20 weeks, diet was increased by $628 \mathrm{~kJ} /$ day (150 kcal/day) [15].

IR was calculated as $\mathrm{U} \mathrm{kg}^{-1}$ day $^{-1}$ (current weight), total insulin requirement (TIR) as U/day and mean blood glucose (MBG) as the weighted mean of SMBG (average of mean CBG before and after main meals, at bedtime and during the night). IR, TIR and MBG were recorded in 1 week intervals from pre-pregnancy to delivery. In the weeks where weight was not available, it was interpolated from preceding and following weeks. The number of episodes of severe hypoglycaemia requiring treatment with glucagon or i.v. glucose was registered.

Pre-pregnancy MBG, IR and TIR were defined as the mean values in the 4 weeks immediately antedating pregnancy.

$\mathrm{HbA}_{1 \mathrm{c}}$ was analysed every 4 weeks and pregnancy measurements were grouped in 4 week periods.

Informed consent The study has the approval of the Institutional Review Board and patients gave oral consent for it.

Statistical analysis Quantitative variables are described as mean \pm standard deviation if normally distributed and as median (range) if not.

Repeated-measures analysis of variance was used to investigate time effect for IR, TIR and MBG with the MIXED procedure of SPSS (v15.0). The restricted maximum likelihood method was used, and the normality for residuals was evaluated with Kolmogorov-Smirnov test. The descriptive variables displayed are the values estimated from the analysis.

Significance was tested for each week vs pre-pregnancy and the previous weeks' results. For this last analysis, prepregnancy levels were compared with those in week 1 and, if non-significant, with those in week 2 and so on. Significance was set at $p$ value $<0.05$ and was corrected according to Bonferroni to $<0.0012$ for MBG, IR and TIR (41 comparisons) and to $<0.005$ for $\mathrm{HbA}_{1 \mathrm{c}}$ analysis (ten comparisons).

Peaks and nadirs were defined as the maximum and minimum values in Fig. 1, provided that a significant modification in the analysis vs previous weeks was present in that period.

In the different periods of insulin increase and decrease, the corresponding $\boldsymbol{\Delta}$ and $\boldsymbol{\Delta}$ per week (slope) were calculated both as absolute and per cent values using a simple regression analysis restricted to the period of interest (IR and TIR as dependent variables and GA as predictor). 
Fig. 1 Mean insulin requirements and self-monitored blood glucose in type 1 diabetic pregnant women. Square, capillary blood glucose; circle, total insulin requirement; triangle, insulin requirements

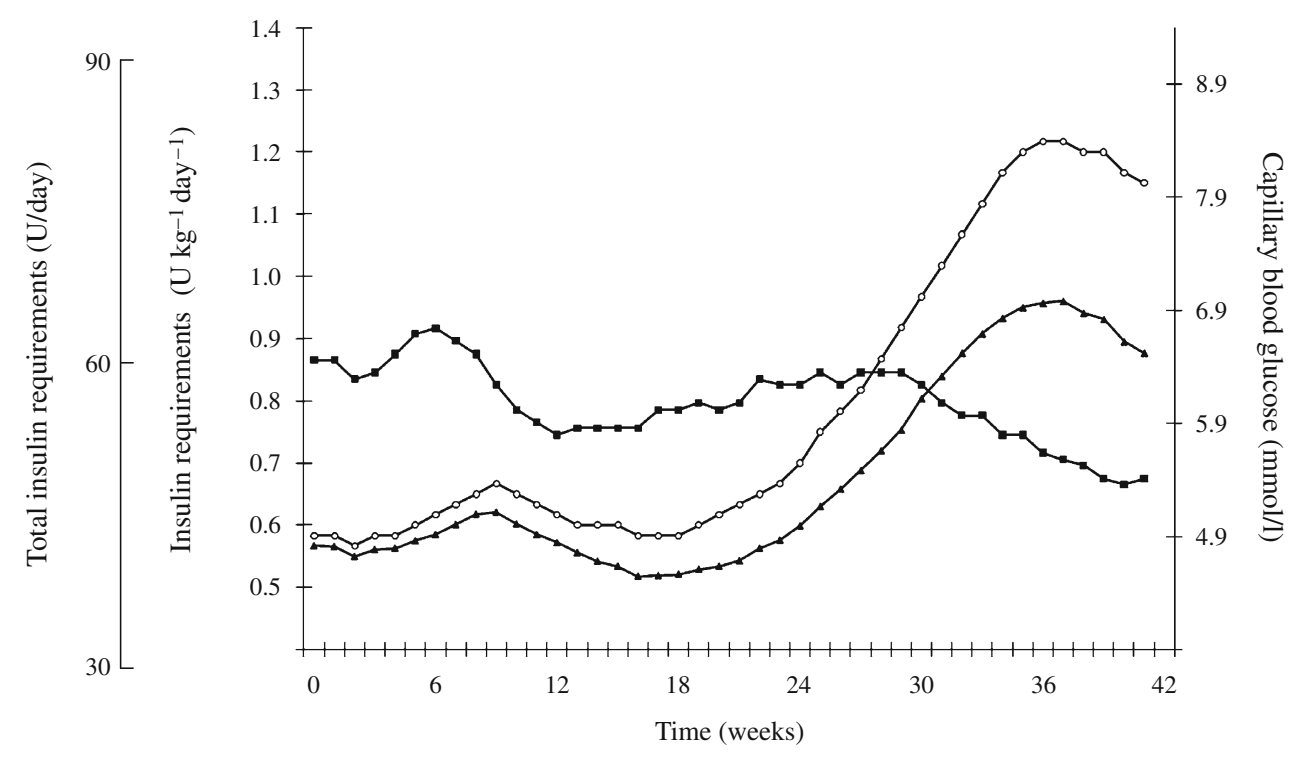

We used two approaches to predict the changes in IR during pregnancy. The first was to construct regression models where the dependent variable was IR and potential predictors were: centred GA defined as GA -21 , (centred GA) ${ }^{2}$, natural log of $\mathrm{GA}$, (natural $\log \mathrm{GA})^{2}$, mean glucose and natural $\log$ of mean glucose; $R^{2}$ and information criteria were taken into account to choose the best model. The second approach was to search for an appropriate polynomial curve (CurveExpert v1.37) to find the best relationships between GA and mean glucose and insulin; the program calculates mean squares of error from the fitted models and identifies as the best model the one with the lowest mean squares of error.

\section{Results}

The study population comprised 65 consecutive women who fulfilled the entry criteria. They delivered between July 1988 and December 2006. The maternal characteristics were: age $30.1 \pm 3.2$ years; BMI $22.8(19.1,34.4) \mathrm{kg} / \mathrm{m}^{2}$; the duration of diabetes $11.8 \pm 7.5$ years; chronic hypertension $1.6 \%$; smokers $23.1 \%$; diabetic retinopathy $26.1 \%$; and established nephropathy $6.1 \%$. Perinatal outcomes were: pregnancy-induced hypertension 9.5\%; delivery week 38 (34-41); preterm birth 13.8\%; Caesarean section 52.3\%; Apgar score $<7$ at $1 \mathrm{~min} 9.5 \%$; large-for-gestational age newborn $33.8 \%$ (using a Spanish growth chart); small-forgestational age newborn 3.1\% (using a Spanish growth chart); major malformations $6.1 \%$; and perinatal mortality $1.5 \%$. All women had near-normal metabolic control since before pregnancy (MBG $6.5 \pm 0.8 \mathrm{mmol} / \mathrm{l}, \mathrm{HbA}_{\mathrm{lc}} 5.67 \pm$ $0.48 \%$ ); The mean IR was $0.667 \pm 0.24 \mathrm{U} \mathrm{kg}^{-1}$ day $^{-1}$ and mean TIR was $40.9 \pm 18.1 \mathrm{U} /$ day.

The MBG during pregnancy ranged from 5.4 to $6.7 \mathrm{mmol} / \mathrm{l}$ (Fig. 1) with a nadir in week 40. Compared with the pre-pregnancy value, MBG during pregnancy was lower in weeks 11 to 16 and 32 to 41 . When compared with previous weeks, the decrease was significant in weeks 11 and 39. The IR ranged from 0.618 to $1.060 \mathrm{U} \mathrm{kg}^{-1}$ day $^{-1}$, and showed more changes and had a higher degree of variation than MBG: a peak in week 9, a nadir in week 16 and a peak in week 37. Compared with the pre-pregnancy IR value, IR was higher in weeks 8 and 9 and from 26 to 41 weeks. When compared with previous weeks, there was an increase in week 8 , a decrease in weeks 11,13 and 16 and increases in week 22 and from week 24 to 34 . The TIR ranged from 40.0 to $79.2 \mathrm{U}$ /day with a pattern of changes similar to that seen for IR, but with a wider variation in values.

The changes in IR and TIR can be expressed as absolute or relative increments and corresponding slopes for a given period (Table 1). IR slopes expressed in percentages provide the best estimation of changes in IR, while TIR slopes best indicate the modifications needed in the insulin dose. The most pronounced slope in per cent values for IR is from week 16 to week 37, followed by the change from week 9 to week 16. The same is true for TIR. However, if we consider the total increment within a period, the major change is that occurring from week 16 to week 37 followed by that from pre-pregnancy to week 9 .

$\mathrm{HbA}_{1 \mathrm{c}}$ ranged from $5.39 \%$ to $5.72 \%$. Compared with the pre-pregnancy value, $\mathrm{HbA}_{1 \mathrm{c}}$ was lower from weeks $13-16$ to weeks 24-28. When comparing values with previous measurements, there was a single decrease at weeks 13 to 16 .

Nine patients $(13.8 \%)$ suffered an episode of severe hypoglycaemia. There were three episodes in week 13 and one episode in weeks 1, 2, 11, 15, 16 and 18. Most episodes occurred in parallel with the decrease in IR.

The best regression model to describe IR was that using centred GA and (centred GA) ${ }^{2}$, which yielded an $R^{2}$ of 
Table 1 Increments and decrements of insulin requirements during pregnancy

\begin{tabular}{|c|c|c|c|c|}
\hline Insulin requirement & $\Delta$ per period & $\Delta$ per week & $\Delta$ per period $(\%)$ & $\Delta$ per week, $\%(95 \% \mathrm{CI})^{\mathrm{a}}$ \\
\hline \multicolumn{5}{|l|}{ IR ( $\mathrm{U} \mathrm{kg}^{-1}$ day $\left.^{-1}\right)$} \\
\hline Pre-pregnancy (9 weeks) & 0.063 & 0.007 & 9.1 & $1.01(0.97,1.05)$ \\
\hline $9-16$ weeks & -0.103 & -0.0147 & -14.3 & $-2.00(-2.02,-1.99)$ \\
\hline 16-37 weeks & 0.442 & 0.0210 & 71.5 & $4.08(4.04,4.12)$ \\
\hline \multicolumn{5}{|l|}{ TIR (U/day) } \\
\hline Pre-pregnancy (9 weeks) & 5.5 & 0.611 & 12.06 & $1.34(1.29,1.38)$ \\
\hline $9-16$ weeks & -5.0 & -0.714 & -10.90 & $-1.51(-1.53,-1.50)$ \\
\hline 16-37 weeks & 38.5 & 1.830 & 94.60 & $5.19(5.13,5.24)$ \\
\hline
\end{tabular}

Periods are defined after the minimum and maximum visual values (Fig. 1)

${ }^{a}$ Derived after regression analysis (see Methods)

0.845. Among all possible linear and non-linear models including a polynomial of up to six degrees, the best-fit program identified the polynomial of fifth degree as the curve best fitting the glucose and insulin data $\left(R^{2} 0.991\right.$ and 0.891 , respectively) (Electronic supplementary material [ESM] Fig. 1).

\section{Discussion}

We have observed that in women with type 1 diabetes mellitus that has been well regulated since before pregnancy, IR and TIR successively displayed significant changes with three significant alterations in direction. The best predictive model was achieved with a polynomial fit of fifth degree.

It is well known that pregnancy is a period of changing IR. The increase in IR in the late second trimester and most of the third trimester is the most characteristic change $[9,11,12]$, followed by the decrease from 10 to 20 weeks $[9,10,16]$ and at term $[9,17,18]$. Less is known about the increase in IR in early pregnancy: Pedersen previously described two cases of diabetic ketoacidosis at 6-8 weeks [19]; Steel et al. reported a single patient having a transient increase in IR at 8-12 weeks [9]; and Jovanovic et al. described an increment between weeks 3 and 7 [16]. In the report of Fuglsang, IR values were relatively flat during the first trimester, but interpretation was difficult because the women did not have near-normal glycaemic control [18]. Data on $\mathrm{MBG}$ are not available from any of the studies listed.

The transient increase in IR reported here cannot be attributed to an attempt to improve metabolic control because women were in strict metabolic control before conception and the increase in IR in early pregnancy was not accompanied by significant changes in $\mathrm{MBG}$ or $\mathrm{HbA}_{1 \mathrm{c}}$. We conclude that a transient increase in IR in early pregnancy has not previously been properly recognised because the IR in early gestation has been studied only very rarely -in a single study in women with good glycaemic regulation [16], and never in connection with MBG.

It is not surprising that in quantitative terms, the most important changes in IR and TIR are those taking place between 16 and 37 weeks, as this is the characteristic IR pattern in pregnancy. Not only is the observed $\Delta$ the largest one, but the slope is the most pronounced. However, MBG was well controlled and the number of episodes of severe hypoglycaemia was small. Conversely, in the first weeks, $\Delta$ and slopes were less pronounced; overall, however, it was a more difficult period: two out of three changes of direction took place in the first 11 weeks and eight out of nine episodes of severe hypoglycaemia occurred in the first 16 weeks. It seems that it is easier to adapt to a continuous large increase in IR than to discrete small changes of differing direction. The apparent decrease in MBG, IR and TIR in the last weeks of pregnancy did not reach significance, although it is a well-recognised phenomenon [9, 17, 18]. We attribute our finding to insufficient statistical power.

For both IR and TIR, the changes in direction are more apparent when compared with previous pregnancy weeks than with the pre-pregnancy value. Changes from requirements in previous weeks best indicate the adaptations needed in daily life. IR values are more relevant from the pathophysiological viewpoint, while TIR values reflect the modifications in insulin dose (number of units) made by the woman/healthcare professional. TIR values show the largest weekly changes (5.19\% vs $4.08 \%$ for IR). Thus, changes in TIR compared with previous weeks best indicate the adaptations of insulin dose in pregnant women with type 1 diabetes. This knowledge can help patients and physicians anticipate the changes in SMBG results.

Some of the participants had a short duration of diabetes with the likely persistence of C-peptide influencing insulin. We assume that if all the women lacked C-peptide, the changes in IR would have been more pronounced. 
As to the underlying mechanisms, the data are better for the increase in IR in the late second trimester and most of the third trimester, with gestational hormones and tumour necrosis factor being responsible [20]. The decrease in IR in the late first trimester has been attributed to decreasing levels of progesterone and thyroid hormones [9]; an increase in C-peptide [21] and morning sickness could potentially have a role [22]. In non-diabetic women, the change in insulin sensitivity at the end of the first trimester depends on BMI; insulin sensitivity decreases at 12-14 weeks in non-obese insulin-sensitive women [23], whereas it increases in obese insulin-resistant women [20, 24]. Similarly, in women with type 1 diabetes mellitus, it has been suggested that the drop in IR at the end of the first trimester is related to BMI [16]. In the current study, an analysis of IR relating to BMI has not been performed because the study was not powered for this analysis. With regard to the transient increase in IR in early pregnancy described here, we can only speculate on the potential mechanisms: levels of chorionic gonadotropin [25], progesterone [25] and thyroid hormones [26] peak in the same period and these hormones can influence IR directly or indirectly. Progesterone decreases insulin sensitivity [27], chorionic gonadotropin has a thyrotropic effect [28] and, in turn, thyroid hormones have a dual effect decreasing insulin sensitivity and increasing insulin clearance [29]. Even though we do not have data about morning sickness in these patients, the increase in insulin requirements coincides with the period when morning sickness is more prominent [22]. As morning sickness is not usually severe and should lead to a decrease in insulin requirements, it is difficult to consider that the association is causal.

We want to stress that the data presented here pertain to the behaviour of the group and that individual patients can diverge from the patterns described either in the magnitude of changes or in the pattern displayed during pregnancy. This has to be taken into account when considering this information in a clinical context.

In conclusion, pregnant women with type 1 diabetes mellitus and tight metabolic control since before pregnancy had changes in IR and TIR with three successive changes of direction. MBG was relatively stable. The sharpest increase in the slope was observed between weeks 16 and 37 but insulin requirements were more unstable in the first 16 weeks. This information could help patients and physicians to react to changing SMBG patterns in a prompt and adequate way.

Acknowledgements We thank the well-regulated patient with type 1 diabetes mellitus who, after becoming pregnant, phoned the clinic about three times per week because of deteriorating glycaemic control and drove our attention to the issue of insulin requirements in early pregnancy. The study was funded by CIBER Bioengineering.
Duality of interest The authors declare that there is no duality of interest associated with this manuscript.

\section{References}

1. Preconception care of women with diabetes (1998) American Diabetes Association: Clinical Practice Recommendations. Diabetes Care 21 (Suppl 1):556-559

2. Guerin A, Nisenbaum R, Ray JG (2007) Use of maternal GHb concentration to estimate the risk of congenital anomalies in the offspring of women with prepregnancy diabetes. Diabetes Care 30:1920-1925

3. Inkster ME, Fahey TP, Donnan PT, Leese GP, Mires GJ, Murphy DJ (2006) Poor glycated haemoglobin control and adverse pregnancy outcomes in type 1 and type 2 diabetes mellitus: systematic review of observational studies. BMC Pregnancy Childbirth 6:30

4. Jensen DM, Korsholm L, Ovesen P et al (2009) Peri-conceptional $\mathrm{A} 1 \mathrm{C}$ and risk of serious adverse pregnancy outcome in 933 women with type 1 diabetes. Diabetes Care 32:1046-1048

5. Hiilesmaa V, Suhonen L, Teramo K (2000) Glycaemic control is associated with pre-eclampsia but not with pregnancy-induced hypertension in women with type I diabetes mellitus. Diabetologia 43:1534-1539

6. Koukkou E, Young P, Lowy C (1997) The effect of maternal glycemic control on fetal growth in diabetic pregnancies. Am J Perinatol 14:547-552

7. Banerjee S, Ghosh US, Banerjee D (2004) Effect of tight glycaemic control on fetal complications in diabetic pregnancies. J Assoc Phys India 52:109-113

8. Ylinen K (1987) High maternal levels of hemoglobin A1c associated with delayed fetal lung maturation in insulindependent diabetic pregnancies. Acta Obstet Gynecol Scand 66:263-266

9. Steel JM, Johnstone FD, Hume R, Mao J-H (1994) Insulin requirements during pregnancy in women with type I diabetes. Obstet Gynecol 83:253-258

10. Kimmerle R, Heinemann L, Delecki A, Berger M (1992) Severe hypoglycemia incidence and predisposing factors in 85 pregnancies of type I diabetic women. Diabetes Care 15:1034-1037

11. Coustan DR, Reece EA, Sherwin RS et al (1986) A randomized clinical trial of the insulin pump vs intensive conventional therapy in diabetic pregnancies. JAMA 255:631-636

12. Langer O, Anyaegbunam A, Brustman L, Guidetti D, Levy J, Mazze R (1988) Pregestational diabetes: Insulin requirements throughout pregnancy. Am J Obstet Gynecol 159:616-621

13. Evers IM, de Valk HW, Visser GH (2004) Risk of complications of pregnancy in women with type 1 diabetes: nationwide prospective study in the Netherlands. BMJ 328:915

14. Harris JA, Benedict FG (1919) A biometric study of basal metabolism in man. Publication no. 279. Carnegie Institution of Washington, Washington

15. Durnin JG (1991) Energy requirements of pregnancy. Diabetes 40 (Suppl 2):152-156

16. Jovanovic L, Knopp RH, Brown Z, National Institute of Child Health and Human Development Diabetes in Early Pregnancy Study Group et al (2001) Declining insulin requirement in the late first trimester of diabetic pregnancy. Diabetes Care 24: $1130-1136$

17. McManus RM, Ryan EA (1992) Insulin requirements in insulindependent and insulin-requiring GDM women during final month of pregnancy. Diabetes Care 15:1323-1327

18. Fuglsang J, Lauszus F, Flyvbjerg A, Ovesen P (2003) Human placental growth hormone, insulin-like growth factor I and -II, and 
insulin requirements during pregnancy in type 1 diabetes. J Clin Endocrinol Metab 88:4355-4361

19. Pedersen J (1981) Influencia del embarazo en la mujer diabética. In: Pedersen J (ed) La gestante diabética y su recién nacido. Salvat Editores, Barcelona, pp 77-87

20. Kirwan JP, Hauguel-De Mouzon S, Lepercq J et al (2002) TNFalpha is a predictor of insulin resistance in human pregnancy. Diabetes 51:2207-2213

21. Nielsen LR, Rehfeld JF, Pedersen-Bjergaard U, Damm P, Mathiesen ER (2009) Pregnancy-induced rise in serum Cpeptide concentrations in women with Type 1 diabetes. Diabetes Care 32:1052-1057

22. Gadsby R, Barnie-Adshead AM, Jagger C (1993) A prospective study of nausea and vomiting during pregnancy. Br J Gen Pract 43:245-248

23. Catalano PM, Roman-Drago NM, Amini SB, Sims EA (1998) Longitudinal changes in body composition and energy balance in lean women with normal and abnormal glucose tolerance during pregnancy. Am J Obstet Gynecol 179:156-165
24. Okereke NC, Huston-Presley L, Amini SB, Kalhan S, Catalano PM (2004) Longitudinal changes in energy expenditure and body composition in obese women with normal and impaired glucose tolerance. Am J Physiol Endocrinol Metab. 287:E472-E479

25. Mishell DR Jr, Thorneycroft IH, Nagata Y, Murata T, Nakamura RM (1973) Serum gonadotropin and steroid patterns in early human gestation. Am J Obstet Gynecol 117:631-642

26. Glinoer D, de Nayer P, Bourdoux P et al (1990) Regulation of maternal thyroid function during pregnancy. J Clin Endocrinol Metab 71:276-287

27. Ryan EA, Enns L (1988) Role of gestational hormones in the induction of insulin resistance. J Clin Endocrinol Metab 67:341-347

28. Kennedy RL, Darne J, Gryffiths H, Price A, Davies R, Cohn M (1990) Thyroid-stimulatory effects of human chorionic gonadotrophin in early pregnancy. Horm Res 33:177-183

29. Ohguni S, Notsu K, Kato Y (1995) Correlation of plasma free thyroxine levels with insulin sensitivity and metabolic clearance rate of insulin in patients with hyperthyroid Graves' disease. Intern Med 34:339-341 